Case presentation We report a 2.5 year-old Caucasian female who presented with sudden onset right- hemiplegia and irritability. She had minor head trauma earlier that day but no loss of consciousness. She had a history of recurrent upper respiratory tract infections, fatigue, poor diet and constipation over the preceding months but no recent illnesses. These symptoms followed a trip to Rwanda where she had a bout of gastroeneteritis. Blood tests were noteworthy for a reduced Haemoglobin of $6.4 \mathrm{~g} / \mathrm{dl}$ with a repeat $\mathrm{Hb}$ of $5.7 \mathrm{~g} / \mathrm{dl}$ (normocytic, normochromic), reduced reticulocyte count of 3.9 and platelet count of 614 but normal liver function. Borellia, Varicella, Mycoplasma and Parvovirus B19 serology were negative

CT Brain was normal. A time-of-flight MR angiography of the circle of Willis showed diffuse ischaemia within the cortex of the left cerebral hemisphere involving the frontal, parietal and occipital lobes. No large vessel occlusion or haemorrhage were seen, and intracranial and extracranial carotid and vertebral arteries were normal. Echo and bubble study was normal.

Abdominal ultrasound showed a calculus in the neck of the gallbladder without cholecystitis. Repeat Parvovirus B19 IgG was positive

Discussion Previous case-control studies have suggested that the risk of childhood arterial ischaemic stroke is increased transiently in the context of acute infection. A multi-centre study in 2017 (Vascular effects of Infection in Paediatric Stroke, VIPS) Study found serological evidence of Parvovirus B19(PVB19) in $6 \%$ of cases of childhood arterial ischaemic stroke. Parvovirus B19 is a DNA virus can cause sub-clinical infection or manifest with flu-like symptoms. Infection is typically mild, but complications can include chronic anaemia.

It has been hypothesised in previous studies that Parvovirus may injure cardiac and arterial endothelium, promoting thrombus or arterial stenosis. However, it is worth noting that neither stenosis nor thrombus were detected in our patient.

Conclusion Parvovirus B19 is an important consideration in cases of stroke in children in particular in the context of retiuculocytopenia and normocytic normochromic anaemia.

\section{P384 MORE THAN MEETS THE EYE}

Joana Figueirinha* , Sara Rolim, Ana Sofia Gomes, Filipa Pinto, Paulo Teixeira. Centro Hospitalar do Médio Ave, Vila Nova de Famalicão, Portugal

\subsection{6/archdischild-2019-epa.730}

Background A teenager with periorbital edema can be a challenging diagnosis since there are numerous diseases that can have this sign, both infectious and non-infectious

Case-report A 14-year-old female presents in the Emergency Department (E.D.) with a bilateral periorbital edema and fatigue that started 5 days before. She denied itchiness, visual complaints, ocular pain or eye discharge. There was no eyelid inflammation and no peripheral edema. Blood tests were normal and proteinuria was excluded. She was sent home with an antihistamine, but came back to the E.D. 3 days later (day 8 of disease) with the same complaint. Apart from the periorbital edema and the fatigue, she had pharyngitis without tonsillar exudate, elevated aminotransferases and lactate dehydrogenase and relative lymphocytosis (52.9\%). The PaulBunnel test was negative and the tests for viral capsular antigens (VCA IgM and IgG) for Epstein-Barr virus (EBV) were negative. She developed fever between day 8 and 10. She was referred to a Pediatric consultation and reevaluated at day 11 .
She still maintained periorbital edema and had a painful palpable liver edge. The abdominal ultrasound showed hepatosplenomegaly. She repeated the blood tests two weeks after and had lymphocytosis (5190/uL; 66.5\%), elevated aminotransferases and lactate dehydrogenase, and she was EBV VCA IgM and IgG positive, which confirmed active infectious mononucleosis. The testing for autoimmune disease, thyroid disease, and human immunodeficiency virus (HIV) and hepatitis viruses were negative.

Conclusion EBV can affect virtually any organ system and periorbital edema can be the first symptom of infectious mononucleosis. This is an atypical case for there was a lack of other more classic symptoms, like sore throat and lymphadenopathy, and the serology was negative at the beginning of the disease. This lead to a more exhaustive investigation in order to exclude autoimmune and thyroid diseases and viral infections like hepatitis and HIV.

\section{P385 PRIMARY CAREGIVERS KNOWLEDGE ON HOME MANAGEMENT OF CHILDHOOD FEVER}

${ }^{1}$ Hemal De silva*, Shreenika De Silva ${ }^{2},{ }^{1}$ Kumudu Weerasekera. ${ }^{1}$ Ministry of Health, Colombo, Sri Lanka; ${ }^{2}$ University of Colombo, Colombo, Sri Lanka

\subsection{6/archdischild-2019-epa.731}

Introduction The objective was to assess the knowledge on childhood fever and knowledge and practices on home management of a febrile child among the primary caregivers; the socio-demographic predictors and the source of information associated with the knowledge and practices.

Methods A descriptive cross-sectional study was carried out in four randomly selected wards at the Lady Ridgeway Hospital Sri Lanka among 400 primary caregivers of children less than 12 years of age admitted with fever. A pre-tested, pre-coded, interviewer administered questionnaire was used.

Results Normal body temperature and temperature during fever were correctly mentioned by $46.2 \%$ and $35.8 \%$ of the study population respectively. Although $52.8 \%$ knew about a diurnal variation of normal body temperature, only $3.5 \%$ described it correctly. Fits were thought to be an effect of fever by $73.8 \%$. However, only $33.5 \%$ mentioned dehydration as an effect. Touching the skin is not different from using the thermometer for identification and monitoring of fever was a belief among $21.9 \%$ of the respondents. Mercury thermometer was identified as the best thermometer by $45.8 \%$, while it was $34 \%$ for digital. Paracetamol was not identified as the best antipyretic by $2.3 \%$. The helpfulness of blood tests before visiting a medical practitioner was mentioned by $48 \%$ of the respondents.

In practice, $60 \%$ of the study population used a thermometer for detection and/or monitoring of fever. The thermometer was used by $54.0 \%$ for identification of fever while $45.8 \%$ used the touch method. In monitoring of fever $59.5 \%$ used the thermometer while $40.5 \%$ used only the touch method.

Paracetamol was used by $99 \%$ of the study population to treat the febrile child at home. Non-Steroidal Anti-Inflammatory Drugs were also used by $1.5 \%$. The dose of paracetamol given to the child was too low for $6.5 \%$ while it was too high for $24.5 \%$. Awakening the sleeping child to administer antipyretics was practiced by $91.8 \%$. Dengue NS1 antigen test was done by $10.5 \%$ and a full blood count by $31.3 \%$ before seeking medical assistance. Statistically significant associations 